International Journal of Bifurcation and Chaos, Vol. 13, No. 11 (2003) 3395-3407

(c) World Scientific Publishing Company

\title{
STEADY-STATE ANALYSIS OF NONLINEARLY COUPLED CHUA'S CIRCUITS WITH PERIODIC INPUT
}

\author{
F. A. SAVACI \\ Electrical and Electronics Engineering Department, \\ Izmir Institute of Technology, \\ Gülbahçe, Urla, Izmir, Turkey \\ savaci@likya.iyte.edu.tr \\ M. E. YALÇIN* \\ Department of Electrical Engineering, ESAT-SISTA, \\ Katholieke Universiteit Leuven, \\ Kasteelpark Arenberg 10, B-3001 Leuven, Belgium \\ mustak.yalcin@esat.kuleuven.ac.be \\ C. GÜZELIŞ \\ Electrical and Electronics Engineering Department, \\ Dokuz Eylül University, \\ Buca, Izmir, Turkey \\ cuneyt.guzelis@eee.deu.edu.tr
}

Received May 9, 2002; Revised June 10, 2002

\begin{abstract}
In this paper, nonlinearly coupled identical Chua's circuits, when driven by sinusoidal signal have been analyzed in the time-domain by using the steady-state analysis techniques of piecewiselinear dynamic systems. With such techniques, it has become possible to obtain analytical expressions for the transfer functions in terms of the circuit parameters. The proposed system under consideration has also been studied by analog simulations of the overall system on a hardware realization using off-the-shelf components as well as by a time-domain analysis of the synchronization error.
\end{abstract}

Keywords: Steady-state analysis; piecewise-linear dynamic systems; Chua's circuit; master-slave system with external input.

\section{Introduction}

Dynamics of coupled identical Chua's circuits [Chua et al., 1986] has been extensively investigated by many researchers [Anishchenko et al., 1995; Kapitaniak \& Chua, 1994; Galais \& Ogarzalek, 1999; Kazantsev \& Nekorkin, 2002]. Synchronization is the most frequently described phenomena in coupled dynamic systems. The phenomenon of synchronization was observed for the first time by Christian Huygens in the 17th century who reported that two clocks hung on the same wall tend to synchronize by nonlinear coupling through the elasticity of the wall. More than two centuries later Rayleigh, Van der Pol, Andronov-Witt and the other scientists [Minorsky, 1962] have developed the theory of this phenomenon. Based on these

*Author for correspondence. 
theoretical frameworks various notions of chaos synchronizations such as generalized synchronization [Afraimovich et al., 1987] complete synchronization [Pecora \& Carroll, 1990], partial synchronization [Maistrenko \& Popovych, 2000] and phase synchronization [Rosenblum et al., 1997] have been developed. The pioneering work [Pecora \& Carroll, 1990], has increased the interest in synchronization after having recently found many applications particularly in telecommunications [Abel \& Schwarz, 2002], in mechanical systems [Blekhman et al., 1995] and in control theory [Nijmeijer, 2001].

Furthermore, synchronization phenomena has also been investigated in periodically forced chaotic systems [Carroll \& Pecora, 1993; Carroll, 1994; Pikovsky et al., 1997; Suykens et al., 1997] and it was shown in [Carroll \& Pecora, 1993] that the synchronizing of chaotic circuits may also be extended to periodically forced circuits by using a feedback device to compensate the zero exponent due to periodic frequency which avoids the synchronization of the response system with the drive system.

In this paper, two coupled identical Chua's circuits which are externally driven [Carroll \& Pecora, 1993] have been investigated in the time-domain by modeling the system as the piecewise-linear dynamic system and using the well-known techniques given in [Hajj \& Skelboe, 1981; Armanazi, 1973], and then the transfer functions between the synchronization error and the input signal have been analytically found.

The proposed system which is composed of two coupled identical Chua's circuits can be viewed as unidirectionally coupled neurons: one of the neurons (first Chua's circuit "the master or drive circuit") is excited by an external sinusoidal input and its output is fed to the other neuron (second Chua's circuit "the slave or response circuit"). The neuron models used here are the same in the sense of chaotic Cellular Neural Networks (CNN [Chua \& Yang, 1988]) introduced in [Güzeliş, 1993] as a third order special case of generalized cellular neural networks of [Güzeliş \& Chua, 1993]. Each neuron with unity self-feedback becomes equivalent to a Chua's circuit [Chua et al., 1986] if it is isolated from the other neuron and from the external input. These neurons are indeed obtained from Chua's circuit by decomposing Chua's diode into a linear positive resistor, a non-linear voltage controlled voltage source and a linear voltage controlled current source. As seen from Fig. 1(a) defining the voltages of the dependent sources as port voltages, a neuron can be considered as a two-port nonlinear dynamical circuit element and also as an input-output system and the output is the voltage of the second port. Such a neural based treatment of Chua's circuit provides:

(i) Obtaining a new hardware realization for Chua's circuit by means of voltage transfer functions synthesis [Yalçın et al., 1996] as an alternative to the known realizations [Kennedy, 1992], and

(ii) Having new ways for excitation and also for nonlinear coupling of Chua's circuits.

The sinusoidal input signal drives to the first Chua's circuit via current source by ensuring the doublescroll regime by choosing the amplitude of the sinusoidal signal sufficiently small and also by adjusting the frequency of the input signal higher than the frequency of the first-harmonic of the limit-cycle of the double-scroll (free or autoperiodic frequency) in the driver and then the output of the first circuit is coupled to the second circuit by means of a dependent current source. Such a choice of the input signal frequency (heteroperiodic or external frequency) is for avoiding the supression of free (autoperiodic) oscillation (and hence the supression of the double-scroll regime) by external frequency [Minorsky, 1962]. Otherwise, the well-known phenomena "frequency entrainment" (i.e. the free oscillation is locked with the frequency of input signal) occurs. Proper choice of the spectrum of the input signal to ensure the chaotic mode of operation has also been investigated in [Pecora \& Carroll, 1991; Murali \& Lakshmanan, 1992].

The feature of the proposed coupled system is that driver and response circuits are not synchronized whenever the driver is excited by a sinusoidal input signal, but synchronization error is an information bearing signal from which the input signal can be recovered.

Section 2 presents the state equations and circuit structure of the proposed coupled system. The system has been analyzed in Sec. 3 by solving the state equations where synchronization errors are state variables and then by finding asymptotical synchronization error between the input and output of the second neuron in terms of the input signal. Analog simulation results obtained in a hardware realization of the system are presented in Sec. 4 . 


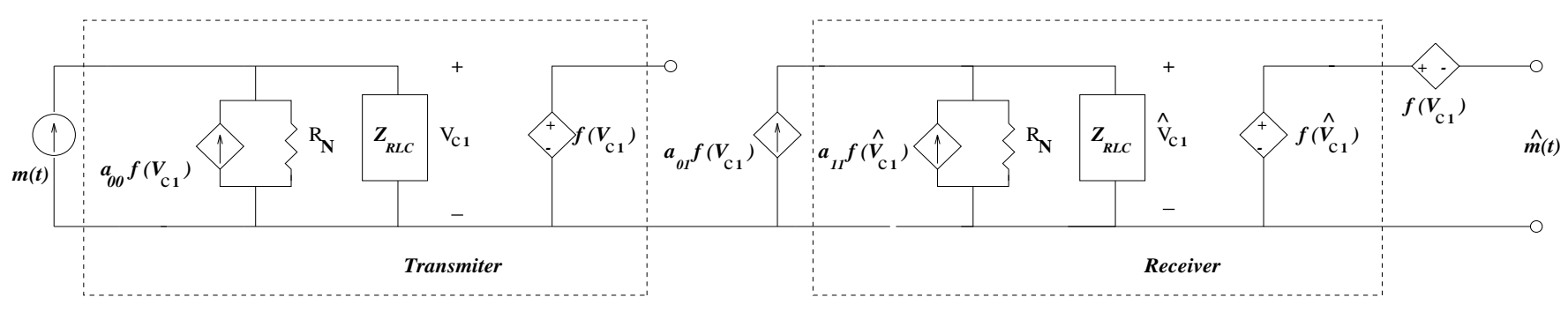

(a)
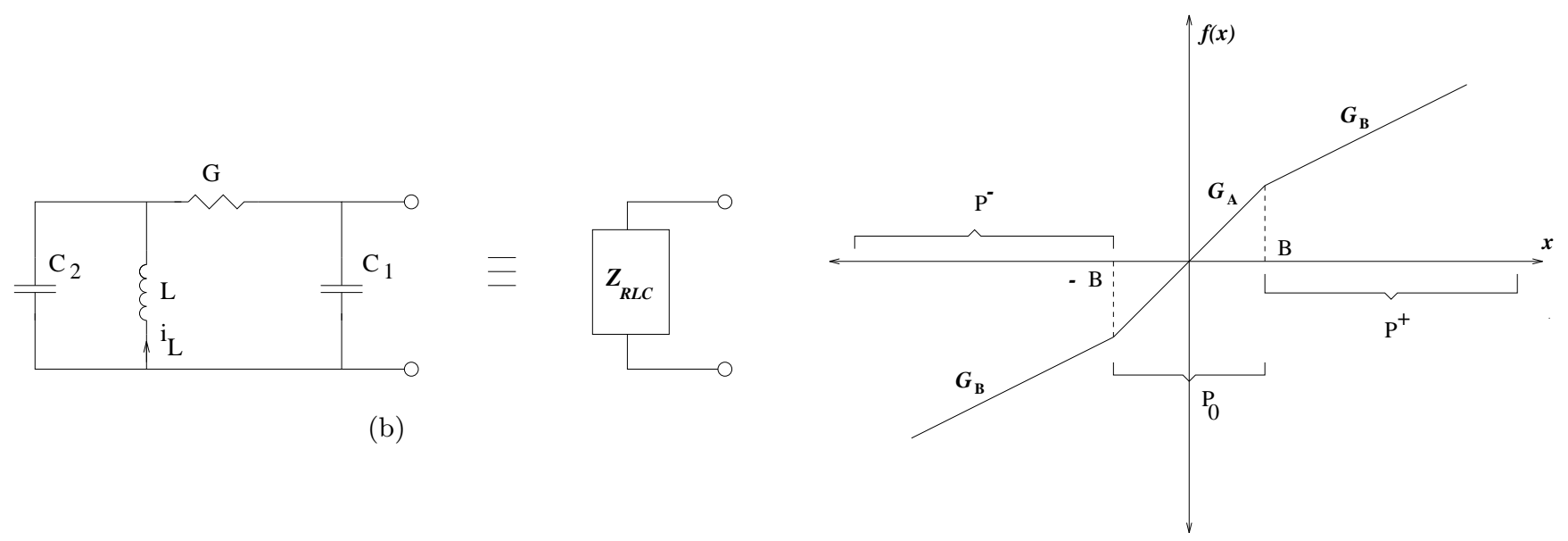

(c)

Fig. 1. (a) Proposed unidirectionally coupled chaotic system. (b) $R, L, C$ equivalent of linear, dynamical 2-terminal element denoted by $Z_{\mathrm{RLC}}$. (c) Piecewise-linear transfer characteristic of voltage-controlled voltage sources.

\section{Time Domain Analysis of the Coupled System}

A circuit realization of the proposed coupled system by using ideal circuit elements is shown in Fig. 1(a). The first neuron circuit is equivalent to original Chua's circuit if transfer characteristics $f(\cdot)$ of nonlinear dependent voltage source are as shown in Fig. 1(c), the self-feedback coefficient is chosen to be unity, and the linear dynamical 2-terminal element $Z_{\mathrm{RLC}}$ is defined as in Fig. 1(b).

The state equations of the proposed coupled system are given as:

\section{The state equations of the first neuron (drive circuit):}

$$
\begin{aligned}
\dot{x}_{1}= & {\left[-(1+\delta) x_{1}+y_{1}+a_{00} f\left(x_{1}\right)\right.} \\
& \left.+a_{10} f\left(x_{2}\right)+m(t)\right] \\
\dot{y}_{1}= & x_{1}-y_{1}+z_{1} \\
\dot{z}_{1}= & -\beta y_{1} .
\end{aligned}
$$

The state equations of the second neuron (response circuit):

$$
\begin{aligned}
\dot{x}_{2}= & \alpha\left[-(1+\delta) x_{2}+y_{2}\right. \\
& \left.+a_{11} f\left(x_{2}\right)+a_{01} f\left(x_{1}\right)\right] \\
\dot{y}_{2}= & \left.\left.x_{2}-y_{2}+z_{2}\right]\right] \\
\dot{z}_{2}= & -\beta y_{2} .
\end{aligned}
$$

where, the piecewise-linear function $f(\cdot)$ with defining $m_{0}=G_{a} / G$ and $m_{1}=G_{b} / G$ can be given as:

$f(x)=m_{1} x+\frac{1}{2}\left(m_{0}-m_{1}\right)(|x+B|-|x-B|)$

In Fig. 1(a) a unidirectionally coupled neuronal system consist of identical Chua's circuits where $a_{10}=0$.

In the sequel, it will be shown that the input signal $m(t)$ can be recovered from the signal $\hat{m}(t)$ which is defined to be the difference between the input $f\left(x_{1}\right)$ and the output $f\left(x_{2}\right)$ of the second neuron as

$$
\hat{m}(t) \stackrel{\text { def }}{=} f\left(x_{1}\right)-f\left(x_{2}\right)
$$


By defining the error vector $\mathbf{E}$ to be the difference of the state vectors of the first neuron $\mathbf{X}_{\mathbf{1}}=\left[\begin{array}{lll}x_{1} & y_{1} & z_{1}\end{array}\right]^{T}$ and the second neuron $\mathbf{X}_{\mathbf{2}}=\left[\begin{array}{lll}x_{2} & y_{2} & z_{2}\end{array}\right]^{T}$ as

$$
\mathbf{E} \stackrel{\text { def }}{=} \mathbf{X}_{1}-\mathbf{X}_{2}
$$

and substracting the state equations of the first neuron from the state equations of the second neuron, the following equations are obtained.

$$
\dot{\mathbf{E}}=\left[\begin{array}{ccc}
-\alpha(1+\delta) & \alpha & 0 \\
1 & -1 & 1 \\
0 & -\beta & 0
\end{array}\right] \mathbf{E}+\left[\begin{array}{c}
\alpha\left(a_{00}-a_{01}\right) \\
0 \\
0
\end{array}\right] f\left(x_{1}\right)+\left[\begin{array}{c}
-\alpha\left(a_{11}-a_{10}\right) \\
0 \\
0
\end{array}\right] f\left(x_{2}\right)+\left[\begin{array}{l}
\alpha \\
0 \\
0
\end{array}\right] m(t)
$$

Using the lemma in [Hajj \& Skelboe, 1981], the following relation can be written

$$
f\left(x_{2}\right)-f\left(x_{1}\right)=s_{k}\left(x_{2}-x_{1}\right)+\mathcal{O}\left(\left|x_{2}-x_{1}\right|\right)
$$

where $s_{k}$ is the slope of $x-f(x)$ characteristics in the region where $x_{1}$ lies, for the time interval $t_{k-1} \leq t \leq t_{k}$, hence $s_{k} \in\left\{m_{0}, m_{1}\right\}$ and the residue term " $\mathcal{O}\left(\left|x_{2}-x_{1}\right|\right)$ " is as

$$
\mathcal{O}\left(\left|x_{2}-x_{1}\right|\right)= \begin{cases}\frac{\left|x_{2}-B\right|}{\left|x_{2}-x_{1}\right|}\left(m_{1}-m_{0}\right)\left(x_{2}-x_{1}\right) & x_{1} \in P_{0}, x_{2} \in P^{+} \\ \frac{\left|x_{2}-B\right|}{\left|x_{2}-x_{1}\right|}\left(m_{0}-m_{1}\right)\left(x_{2}-x_{1}\right) & x_{1} \in P^{+}, x_{2} \in P_{0} \\ \frac{\left|x_{2}-(-B)\right|}{\left|x_{2}-x_{1}\right|}\left(m_{1}-m_{0}\right)\left(x_{2}-x_{1}\right) & x_{1} \in P_{0}, x_{2} \in P^{-} \\ \frac{\left|x_{2}-(-B)\right|}{\left|x_{2}-x_{1}\right|}\left(m_{0}-m_{1}\right)\left(x_{2}-x_{1}\right) & x_{1} \in P^{-}, x_{2} \in P_{0} \\ \left(\frac{\left|x_{2}-(-B)\right|}{\left|x_{2}-x_{1}\right|}-\frac{\left|x_{2}-B\right|}{\left|x_{2}-x_{1}\right|}\right)\left(m_{0}-m_{1}\right)\left(x_{2}-x_{1}\right) & x_{1} \in P^{-}, x_{2} \in P^{+} \\ \left(\frac{\left|x_{2}-(-B)\right|}{\left|x_{2}-x_{1}\right|}-\frac{\left|x_{2}-B\right|}{\left|x_{2}-x_{1}\right|}\right)\left(m_{1}-m_{0}\right)\left(x_{2}-x_{1}\right) & x_{1} \in P^{+}, x_{2} \in P^{-} \\ 0 & x_{1} \text { and } x_{2} \text { in the same region }\end{cases}
$$

where the regions $P^{0}, P^{+}$and $P^{-}$have been shown in Fig. 1(c).

This residue term is nonzero only when $x_{1}$ and $x_{2}$ are at different regions and its magnitude approaches to zero whenever $\left|x_{2}-x_{1}\right|$ approaches to zero. If $x_{2}(t)$ (and/or $x_{1}(t)$ ) is rapidly changing then the probability that $x_{1}$ and $x_{2}$ are placed at different regions will be very high and this nonzero residue term will frequently occur.

By substituting the relation (11) into (10), the following state equations defined for each time interval $T_{k} \stackrel{\text { def }}{=}\left[t_{k-1} t_{k}\right]$ have been obtained as:

$$
\dot{\mathbf{E}}=\left[\begin{array}{ccc}
-\alpha\left[(1+\delta)+\left(a_{00}-a_{11}\right) s_{k}\right] & \alpha & 0 \\
1 & -1 & 1 \\
0 & -\beta & 0
\end{array}\right] \mathbf{E}+\left[\begin{array}{c}
\alpha\left(a_{00}-a_{01}-a_{11}+a_{10}\right) \\
0 \\
0
\end{array}\right] f\left(x_{1}\right)+\mathbf{b}_{\mathbf{N}} \mathcal{O}\left(\left|x_{2}-x_{1}\right|\right)+\mathbf{b} m(t)
$$

where

$$
\mathbf{b}=\left[\begin{array}{lll}
\alpha & 0 & 0
\end{array}\right]^{T}, \quad \mathbf{b}_{\mathbf{N}}=\left[\begin{array}{lll}
-\alpha\left(a_{11}-a_{10}\right) & 0 & 0
\end{array}\right]^{T} .
$$

By choosing the connection weights to satisfy the relation $a_{00}-a_{01}-a_{11}-a_{10}=0$ without loss of a generality, Eq. (12) is further simplified as

$$
\dot{\mathbf{E}}=\mathbf{A}_{k} \mathbf{E}+\mathbf{b} m(t)+\mathbf{b}_{\mathbf{N}} \mathcal{O}\left(\left|x_{2}-x_{1}\right|\right) \text { for } t \in T_{k}
$$


where $\mathbf{A}_{k}$ is a time-varying state matrix

$$
\mathbf{A}_{k}=\left[\begin{array}{ccc}
-\alpha\left[(1+\delta)+\left(a_{00}-a_{11}\right) s_{k}\right] & \alpha & 0 \\
1 & -1 & 1 \\
0 & -\beta & 0
\end{array}\right]
$$

which is equal to strictly Hurwitz constant matrix $\mathbf{A}\left(m_{0}\right)$ (and respectively $\mathbf{A}\left(m_{1}\right)$ ) for the times whenever $x_{1} \in P_{0}$ (and respectively for the times when $\left.x_{1} \in P^{+} \cup P^{-}\right)$.

The state transition matrix of the time-varying state equation system of

$$
\dot{\mathbf{E}}=\mathbf{A}_{k} \mathbf{E} \quad t \in T_{k}
$$

can be obtained using the approach given in [Armanazi, 1973] as:

$\Phi(t, 0)=e^{\mathbf{A}_{n}\left(t-t_{n-1}\right)} e^{\mathbf{A}_{n-1} h_{n-1}}, \ldots, e^{\mathbf{A}_{1} h_{1}}$ for $t \in T_{n}$

where $h_{j} \stackrel{\text { def }}{=} t_{j}-t_{j-1}$ for $j \in\{1,2, \ldots, n-1\}$ and $t_{0}=0$.

Since each of $\mathbf{A}_{k}$ matrices is either $\mathbf{A}\left(m_{0}\right)$ or $\mathbf{A}\left(m_{1}\right)$ both of which are strictly Hurwitz matrices, then there exists a finite number $K$, such that $\|\Phi(t, 0)\| \leq K<\infty, \forall t$. This is a consequence of the fact that each $\left\|e^{\mathbf{A}_{k}\left(t-t_{0}\right)}\right\| \leq N_{k} e^{\alpha_{k}\left(t-t_{0}\right)}$ for some $N_{k}>0, \alpha_{k}<0 \forall t>t_{0}$ and then

$\|\Phi(t, 0)\|$

$$
\begin{array}{r}
\leq\left(\prod_{i=1}^{n} N_{i}\right) e^{\left(\alpha_{n}\left(t-t_{n-1}\right)+\alpha_{n-1} h_{n-1}+\cdots+\alpha_{1} h_{1}\right)} \\
t \in T_{n} .
\end{array}
$$

When the time increases indefinitely, the sum $\alpha_{n}(t-$ $\left.t_{n-1}\right)+\alpha_{n-1} h_{n-1}+\cdots+\alpha_{1} h_{1}$ tends to minus infinity and then $\lim _{t \rightarrow \infty}\|\Phi(t, 0)\|=0$.

The complete solution of (12) for the times $t_{n-1}<t<t_{n}$ is

$$
\begin{aligned}
\mathbf{E}(t)= & \Phi(t, 0) \mathbf{E}(0)+\int_{0}^{t} \Phi(t, \tau) \mathbf{b} m(\tau) d \tau \\
& +\int_{0}^{t} \Phi(t, \tau) \mathbf{b}_{N} \mathcal{O}\left(\left|x_{2}(\tau)-x_{1}(\tau)\right|\right) d \tau
\end{aligned}
$$

The first term will approach zero when $t$ approaches to infinity. The forced solution due to $\mathcal{O}\left(\left|x_{2}-x_{1}\right|\right)$ can be neglected since the occurrence of the nonzero residue term $\left|\mathcal{O}\left(\left|x_{2}-x_{1}\right|\right)\right|$ is not frequent. This is because the probability of $x_{1}$ and $x_{2}$ being at the different regions is small since $x_{1}$ and $x_{2}$ are slowly changing as a result of the assumption that the input signal frequency is very high compared to the fundamental frequency of the limit-cycle of the double-scroll regime. The forced solution term of (17) $\mathbf{E}_{\text {forced }}(t)$ due to $m(t)$ can be found as

$$
\begin{aligned}
\mathbf{E}_{\mathbf{f o r c e d}}(t) & \\
= & \mathbf{E}_{\mathbf{p}_{\mathbf{n}}}(t)-e^{\mathbf{A}_{n}\left(t-t_{n-1}\right)} e^{\mathbf{A}_{n-1} h_{n-1}}, \ldots, e^{\mathbf{A}_{1} h_{1}} \mathbf{E}_{\mathbf{p}_{\mathbf{1}}}(0) \\
& +e^{\mathbf{A}_{n}\left(t-t_{n-1}\right)} e^{\mathbf{A}_{n-1} h_{n-1}}, \ldots, e^{\mathbf{A}_{2} h_{2}}\left[\mathbf{E}_{\mathbf{p}_{\mathbf{1}}}\left(t_{1}\right)-\mathbf{E}_{\mathbf{p}_{\mathbf{2}}}\left(t_{1}\right)\right] \\
& +e^{\mathbf{A}_{n}\left(t-t_{n-1}\right)} e^{\mathbf{A}_{n-1} h_{n-1}}, \ldots, e^{\mathbf{A}_{3} h_{3}}\left[\mathbf{E}_{\mathbf{p}_{\mathbf{2}}}\left(t_{2}\right)-\mathbf{E}_{\mathbf{p}_{\mathbf{3}}}\left(t_{2}\right)\right] \\
& +\cdots+e^{\mathbf{A}_{n}\left(t-t_{n-1}\right)}\left[\mathbf{E}_{\mathbf{p}_{\mathbf{n}-\mathbf{1}}}\left(t_{n-1}\right)-\mathbf{E}_{\mathbf{p}_{\mathbf{n}}}\left(t_{n-1}\right)\right]
\end{aligned}
$$

where $\mathbf{E}_{\mathbf{p}_{\mathbf{k}}}(t)$ is the particular solution during the time interval $T_{k}$ for the truncated state equation system of

$$
\dot{\mathbf{E}}_{\mathbf{p k}}=\mathbf{A}_{k} \mathbf{E}_{\mathbf{p k}}+\mathbf{b} m(t)
$$

due to a sinusoidal input signal $m(t)=M \operatorname{Cos}(w t)$.

The expression (18) has been obtained by an interval-wise application of the relation which gives the forced solution to a linear system in terms of its particular solution and state transition matrix, and by imposing the final value at the present interval on the linear state equations of the next interval as the initial condition.

The particular solution, $\mathbf{E}_{\mathbf{p}_{\mathbf{k}}}(t)$, is an approximate particular solution of (13) since the correct particular solution $\mathbf{E}_{\mathbf{p c}_{\mathbf{k}}}$ should satisfy

$$
\begin{aligned}
\dot{\mathbf{E}}_{\mathbf{p c}_{\mathbf{k}}}= & A\left(s_{k}\right) \mathbf{E}_{\mathbf{p c}_{\mathbf{k}}}+\mathbf{b} m(t) \\
& +\mathcal{O}\left(\left|x_{2_{p c_{k}}}-x_{1_{p c_{k}}}\right|\right) .
\end{aligned}
$$

The approximate particular solution is found as

$$
\mathbf{E}_{\mathbf{p}_{\mathbf{k}}}(t)=\left[\begin{array}{l}
\varepsilon_{1}\left(w, s_{k}\right) \operatorname{Cos}\left(w t+\theta_{1}\left(w, s_{k}\right)\right) \\
\varepsilon_{2}\left(w, s_{k}\right) \operatorname{Cos}\left(w t+\theta_{2}\left(w, s_{k}\right)\right) \\
\varepsilon_{3}\left(w, s_{k}\right) \operatorname{Cos}\left(w t+\theta_{3}\left(w, s_{k}\right)\right)
\end{array}\right] \quad t \in T_{k}
$$

The amplitude and phase of the first component are given for $\delta=1$ as in (22) and (23) while the others which are not related to $\hat{m}(t)$, are omitted here.

$$
\begin{gathered}
\varepsilon_{1}\left(w, s_{k}\right)=M \frac{\sqrt{\varsigma_{1}^{2}\left(w, s_{k}\right)+\varsigma_{2}^{2}\left(w, s_{k}\right)}}{\zeta\left(w, s_{k}\right)} \\
\theta_{1}\left(w, s_{k}\right)=\arctan \left(\frac{\varsigma_{2}\left(w, s_{k}\right)}{\varsigma_{1}\left(w, s_{k}\right)}\right)
\end{gathered}
$$

where

$$
\begin{aligned}
a_{s_{k}}= & -\alpha\left(1+\delta+s_{k}\left(a_{00}-a_{11}\right)\right) \\
\varsigma_{1}\left(w, s_{k}\right)= & -\alpha\left[a_{s_{k}} w^{4}+\left(2 \beta a_{s_{k}}+a_{s_{k}}+\alpha\right) w^{2}\right. \\
& \left.+\left(a_{s_{k}} \beta+\alpha \beta\right) w+a_{s_{k}} \beta^{2}\right], \\
\varsigma_{2}\left(w, s_{k}\right)= & w \alpha\left[w^{4}+(-2 \beta+1+\alpha) w^{2}\right. \\
& \left.-2 \beta w+\beta^{2}-\beta \alpha\right]
\end{aligned}
$$




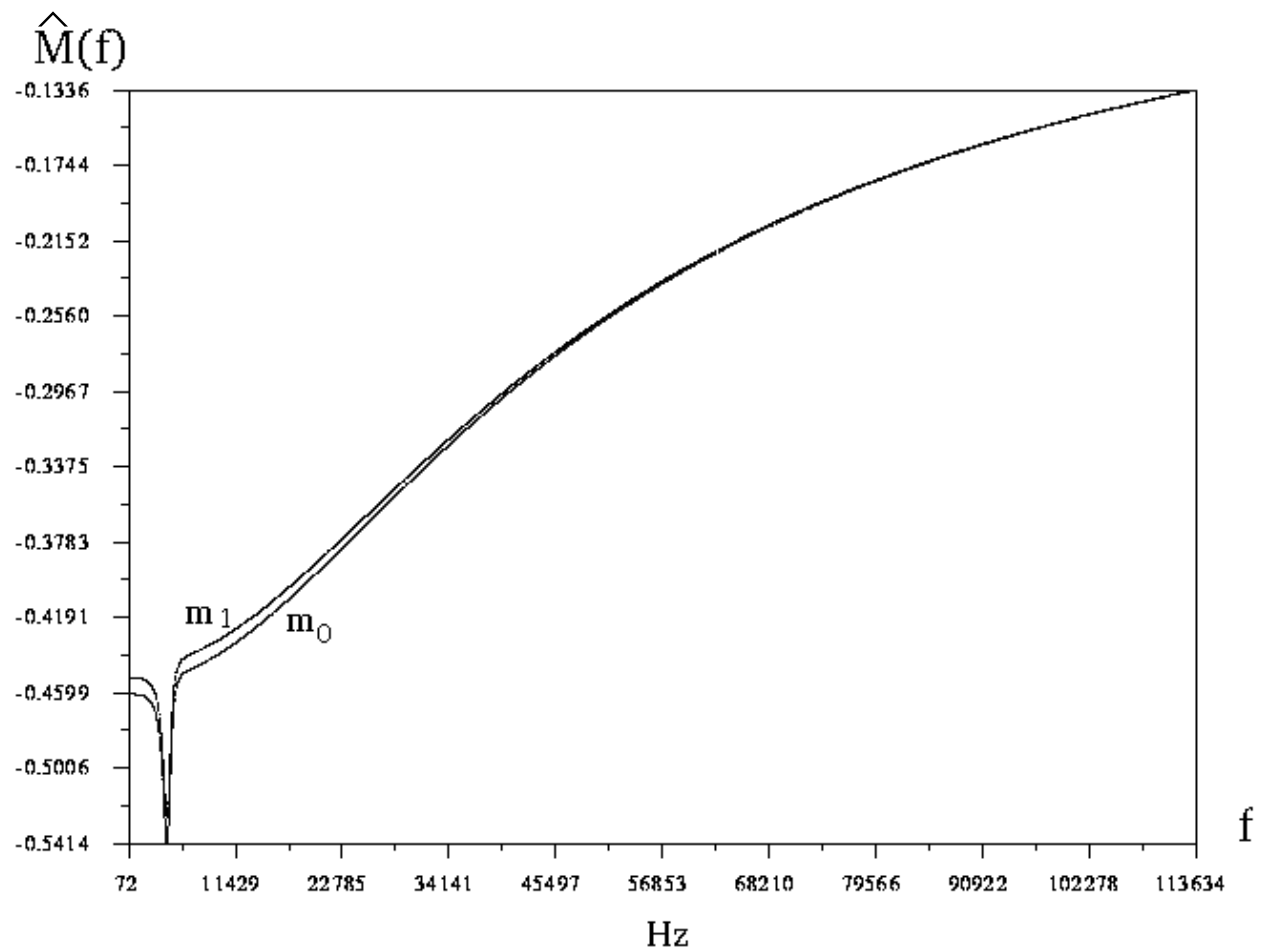

Fig. 2. $\hat{M}(f)$ magnitude spectrum for unity input amplitude, i.e. $M=1$.

and

$$
\begin{aligned}
\zeta\left(w, s_{k}\right)= & -w^{6}+\left(2 \beta-2 \alpha-a_{s_{k}}-1\right) w^{4}+\beta w^{3} \\
& +\left(\beta\left[-\beta+2\left(a_{s_{k}}+\alpha\right)\right]-\left[a_{s_{k}}+\alpha\right]^{2}\right) w^{2} \\
& +\left(-\beta a_{s_{k}} \alpha-\beta a_{s_{k}}\right) w-\beta^{2} a_{s_{k}}^{2} .
\end{aligned}
$$

The forced solution in (18) is approximately equal to $\mathbf{E}_{\mathbf{p}_{\mathbf{n}}}(t)$ at the steady-state since the vectors $\left[\mathbf{E}_{\mathbf{p}_{\mathbf{n}-\mathbf{1}}}\left(t_{n-1}\right)-\mathbf{E}_{\mathbf{p}_{\mathbf{n}}}\left(t_{n-1}\right)\right]$ are almost equal to zero vector which can be seen from the closeness of the characteristics in Figs. 3 and 4 obtained for different slopes $m_{0}, m_{1}$, and since matrix exponentials multiplying these small constant vectors are decreasing with time $t$.

The recovered signal $\hat{m}(t)$ defined in (8) at the time interval $T_{k}$ is

$$
\hat{m}(t)=s_{k} \mathbf{C}^{T} \mathbf{E}(t)+\mathcal{O}\left(\left|x_{2}-x_{1}\right|\right)
$$

with

$$
C=\left[\begin{array}{lll}
1 & 0 & 0
\end{array}\right]^{T} \quad t \in T_{k}
$$

and at the steady-state $\hat{m}(t) \cong s_{k} \mathbf{C}^{T} \mathbf{E}_{\mathbf{p}_{\mathbf{k}}}(t)$ since the forced solution due to $m(t)$ is approximately equal to the particular solution $\mathbf{E}_{\mathbf{p}_{\mathbf{k}}}(t)$ at the steady-state.

Hence, using (22) and (23) the recovered signal can be obtained as:

$$
\hat{m}(t) \cong s_{k} \varepsilon_{1}\left(w, s_{k}\right) \operatorname{Cos}\left(w t+\theta_{1}\left(w, s_{k}\right)\right)
$$

$\hat{M}(w) \stackrel{\text { def }}{=} \varepsilon_{1}\left(w, s_{k}\right)$ the magnitude and $\hat{\theta}(w) \stackrel{\text { def }}{=}$ $\theta_{1}\left(w, s_{k}\right)$ the phase characteristics for a frequency range, where double-scroll regime has been observed to be preserved (see Sec. 4), are given for both $m_{0}$ and $m_{1}$ in Figs. 2 and 3 where $a_{00}=1, a_{11}=0.1$, $a_{01}=0.9, a_{10}=0$.

The critical frequency $f_{c}=4.3 \mathrm{kHz}$ around which the characteristics fail to be flat is the frequency closest to the natural frequencies of (19). In other words, as $f$ (external input frequency) approaches to $f_{c}$, the particular solutions of (19) become undefined.

It should be noted that the overall system behaves like a filter having the characteristics in Figs. 3 and 4. Their unwanted effects on the input signal can be compensated by a post filter. As observed from analog simulations, for the frequency range considered in the above figures, the double scroll regimes of the first neuron and second neuron Chua's circuits are preserved. This means that an input signal having upto $\approx 40 \mathrm{kHz}$ bandwidth might be recovered well by the proposed system. Input signals, which have frequencies falling out of the frequency range, can be brought into this region by using a sinusoidal carrier signal. 


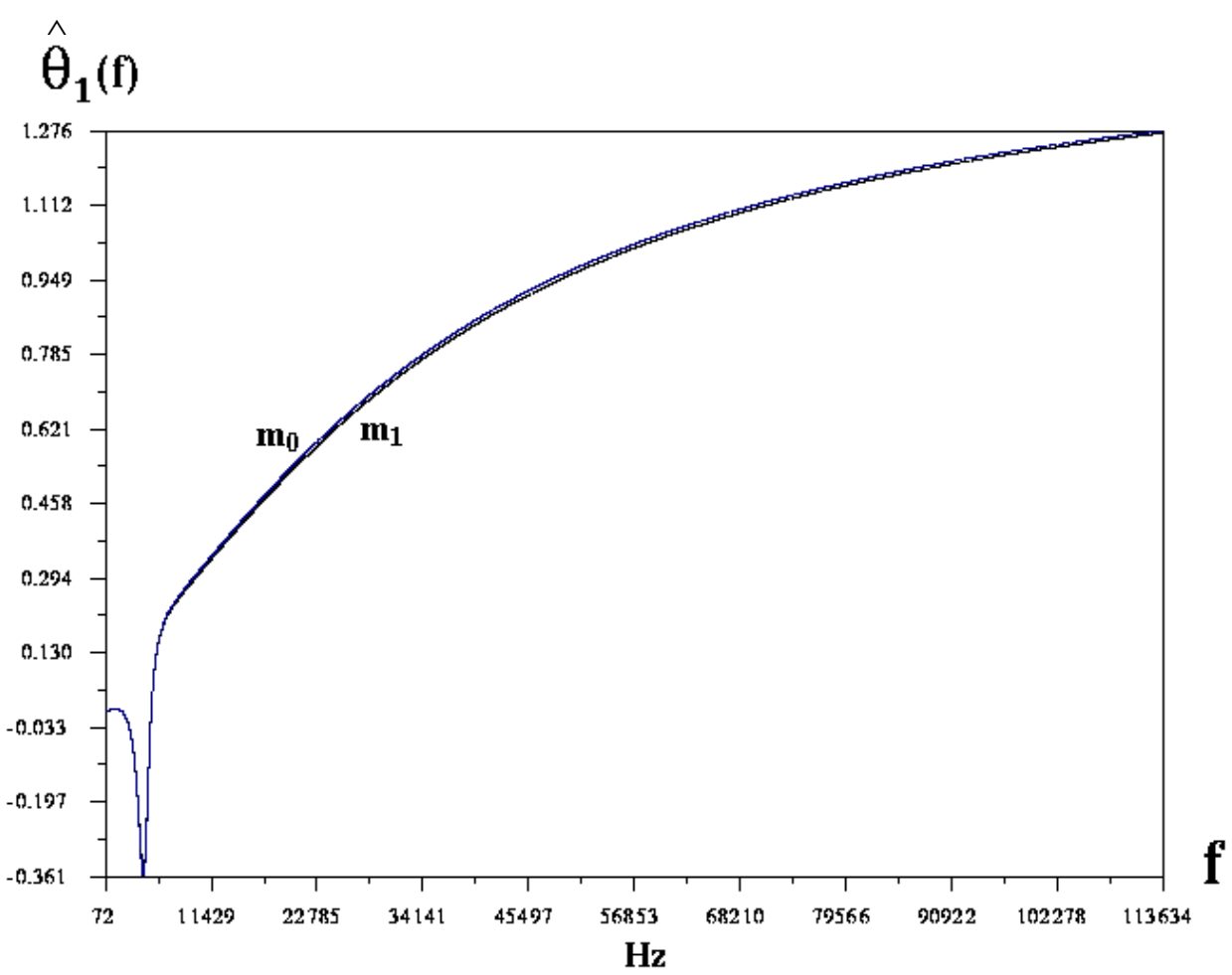

Fig. 3. $\hat{\theta}(f)$ phase spectrum for zero message phase.

\section{Hardware Realization}

In this section, the time domain analysis has been confirmed by experimental observations done on a hardware realization. In the realization, off-the-shelf $R, L, C$ components with $C_{1}=1 \mathrm{nF}, C_{2}=22 \mathrm{nF}$, $R=10 \mathrm{k} \Omega, L=68 \mathrm{mH}, R_{N}=10 \mathrm{k} \Omega$ and the op amps TLC-082 for the synthesis of dependent sources based on the voltage controlled voltage source approach [Yalçın et al., 1996] have been used for both the first neuron and second neuron Chua's circuits. For a large $f(x)$ signal to be transferred and also for a voltage transfer characteristic $x-f(x)$ that is easy to implement by op amps, firstly, voltage dependent sources have been realized for the slopes $\bar{G}_{A}=15 / 7$ and $\bar{G}_{B}=12 / 7$, and then, the resulting scaled $f(x)$ has been applied to voltage controlled current sources with a gain factor $10^{-4}$; resulting in a characteristic having the slopes $G_{A}=\frac{15}{7} 10^{-4}$ and $G_{B}=\frac{12}{7} 10^{-4}$ to obtain double-scroll regime. This indicates an implementation advantage of the neural based treatment of Chua's circuit.

The current $m(t)$ driving the first neuron has been obtained as the current of a voltage controlled current source with a gain factor $10^{-5}$ mho such that the peak to peak value of the control volt- age $V_{m}$ is in the interval of $[2 \mathrm{~V}, 11 \mathrm{~V}]$. It has been observed that excitation by such an input signal $m(t)$ having the frequency range $[4 \mathrm{kHz}, 100 \mathrm{kHz}]$ does not destroy the double-scroll regime of the first neuron.

The $V_{C 1}-V_{C 2}$ plot for the first neuron driven by the source with $V_{m}$ of $10 \mathrm{~V}$ peak to peak value and with $f=17 \mathrm{kHz}$; and the $\hat{V}_{C 1}-\hat{V}_{C 2}$ plot for the second neuron are given in Figs. 4(a) and 4(b).

The waveforms of the source $V_{m}(t)$ with the abovementioned amplitude and frequency, the transmitted signal, i.e. $10^{4} f\left(V_{C 1}(t)\right)$, and the recovered signal $\hat{m}(t)$ are given in Figs. 5(a) and 5(b).

As mentioned before, the input signal can be recovered by using the synchronization error. This fact can be seen from the following " $V_{C 1}(t)$ versus $\hat{V}_{C 1}(t)$ " and " $V_{m}(t)$ versus $\hat{m}(t)$ ". Figure 6(a) shows the synchronization error and Fig. 6(b) indicates the phase delay between $m(t)$ and $\hat{m}(t)$.

In order to examine the robustness of the proposed system against channel noise, a $(0-100 \mathrm{kHz})$ white-noise with $0.3 \mathrm{~V}$ peak to peak value which is supplied by an analog noise generator is added to the transmitting signal. The observed waveform for the recovered signal $\hat{m}(t)$ together with the original is given in Fig. 7. Considering $13 \mathrm{~V}$ peak 


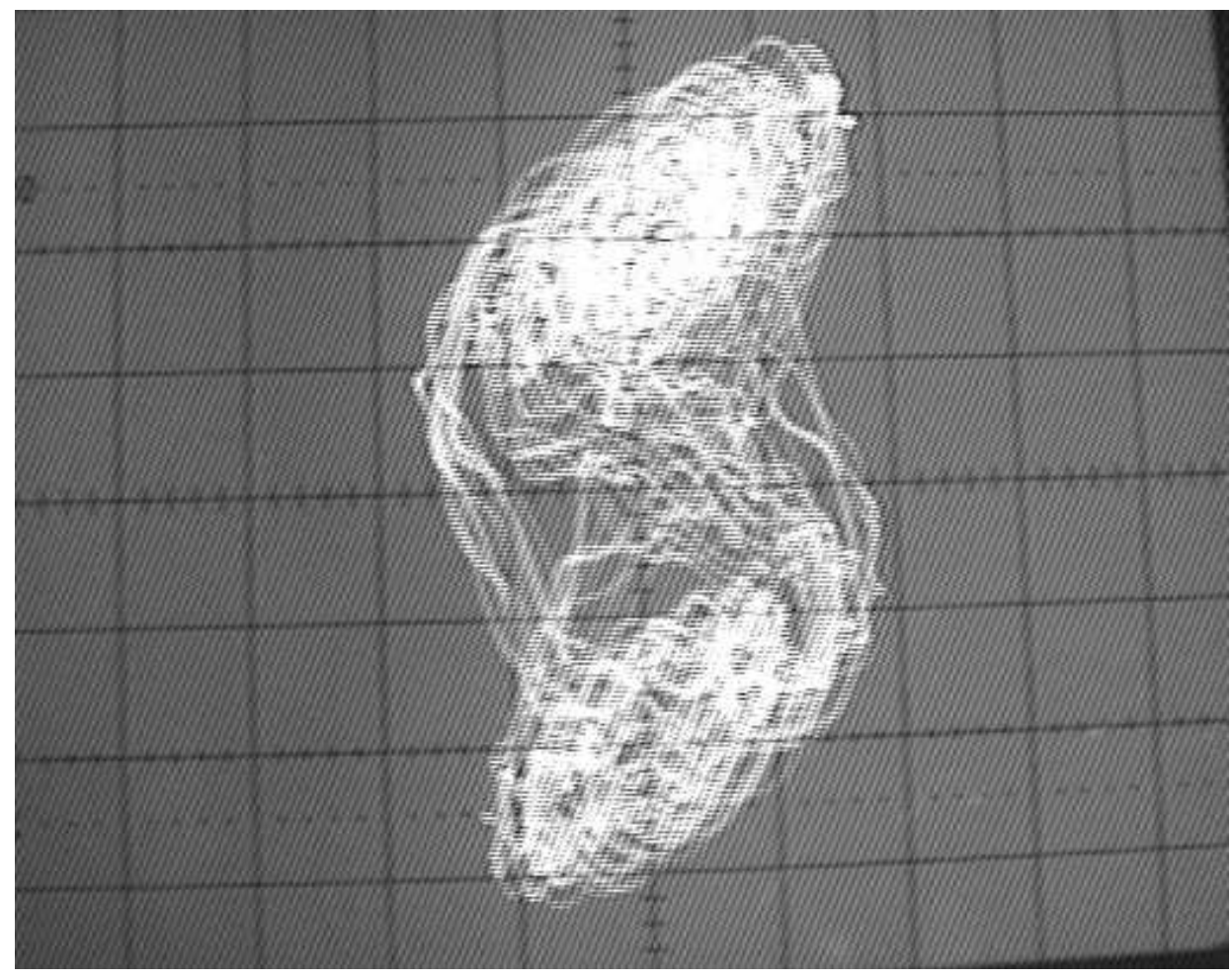

(a)

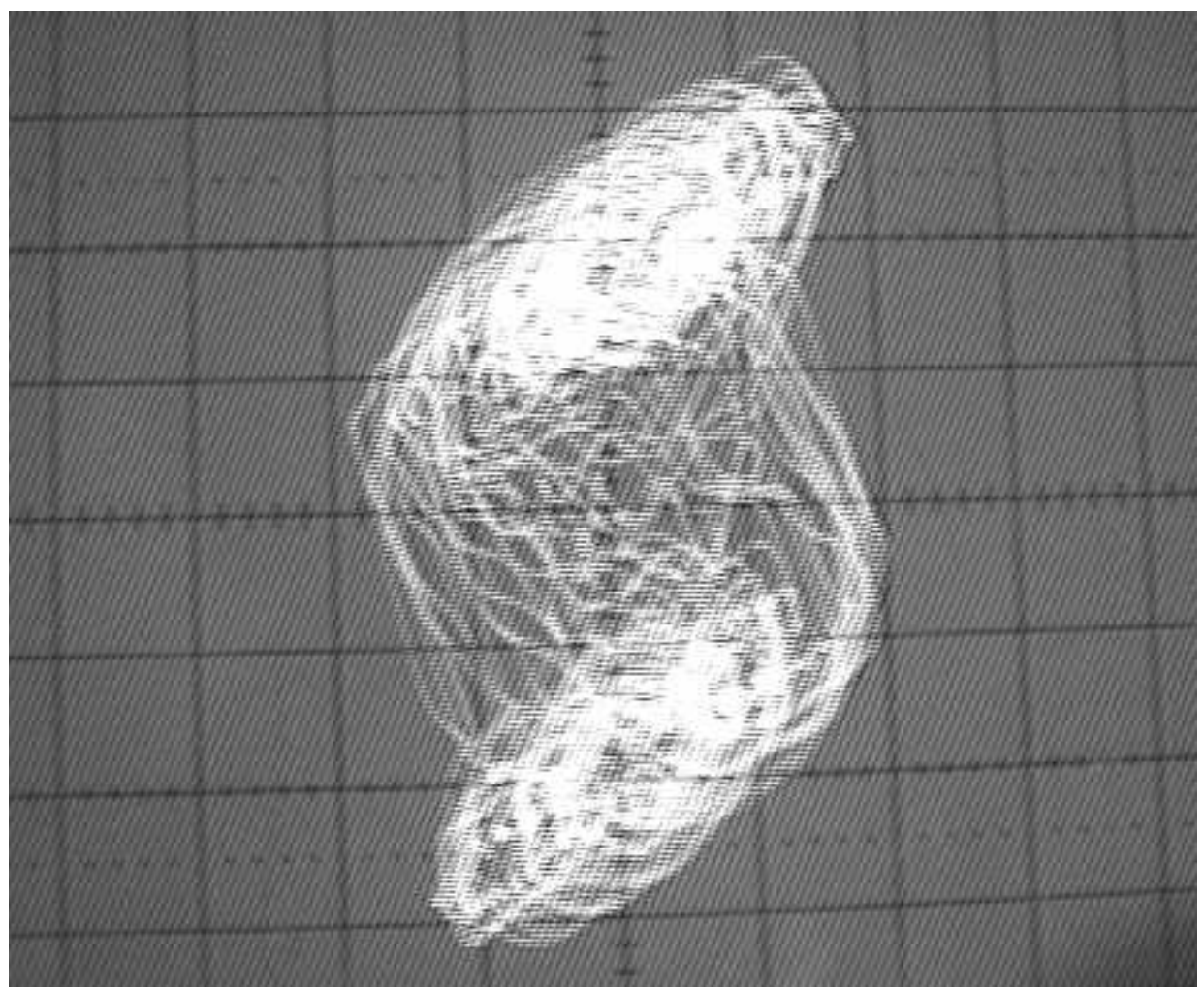

(b)

Fig. 4. (a) $V_{C 1}-V_{C 2}$ plot for the first neuron $\left(1 \mathrm{~V} / \operatorname{div}-0.2 \mathrm{~V} /\right.$ div). (b) $\hat{V}_{C 1}-\hat{V}_{C 2}$ plot for the second neuron $(1 \mathrm{~V} /$ div$0.2 \mathrm{~V} / \operatorname{div})$. 


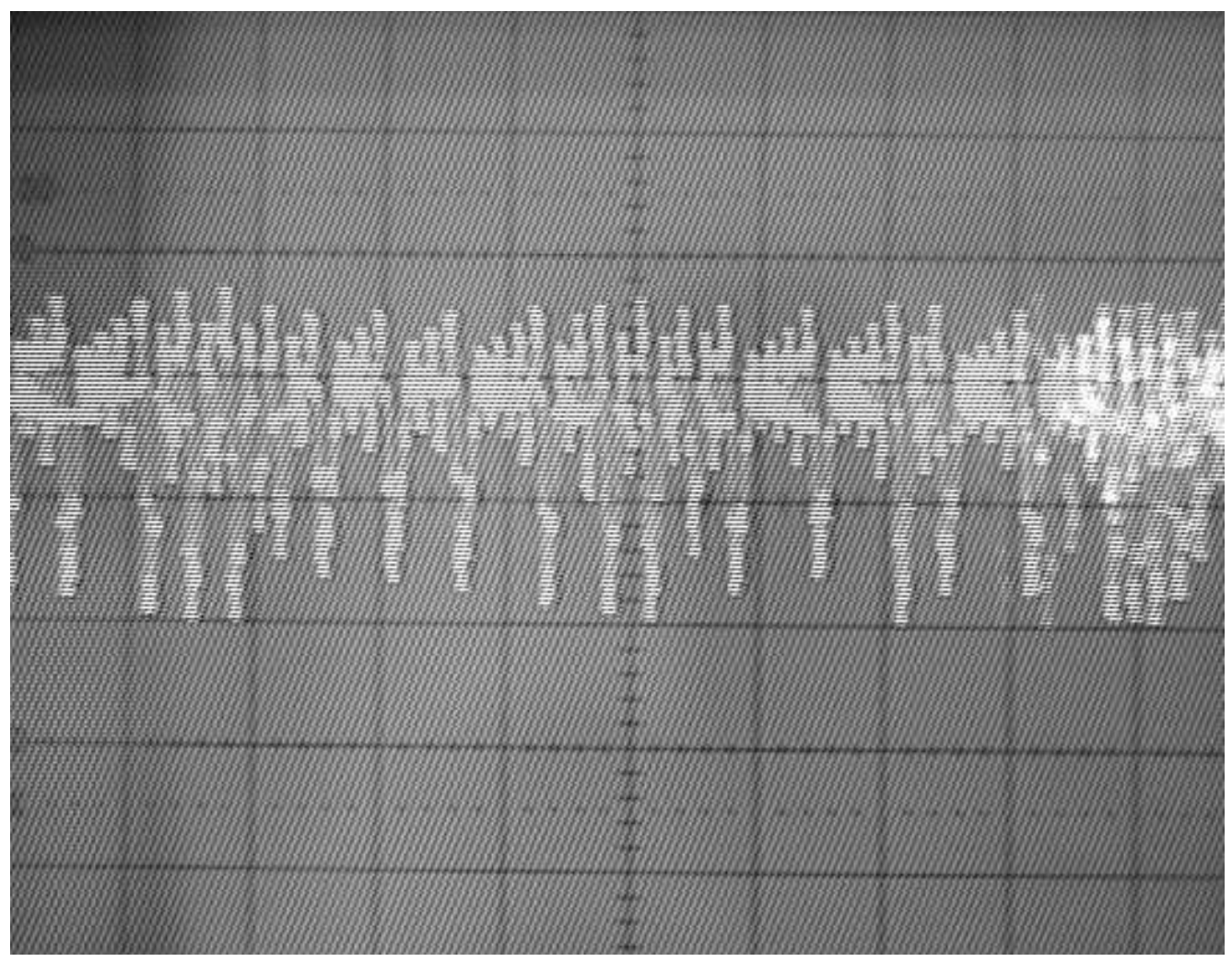

(a)

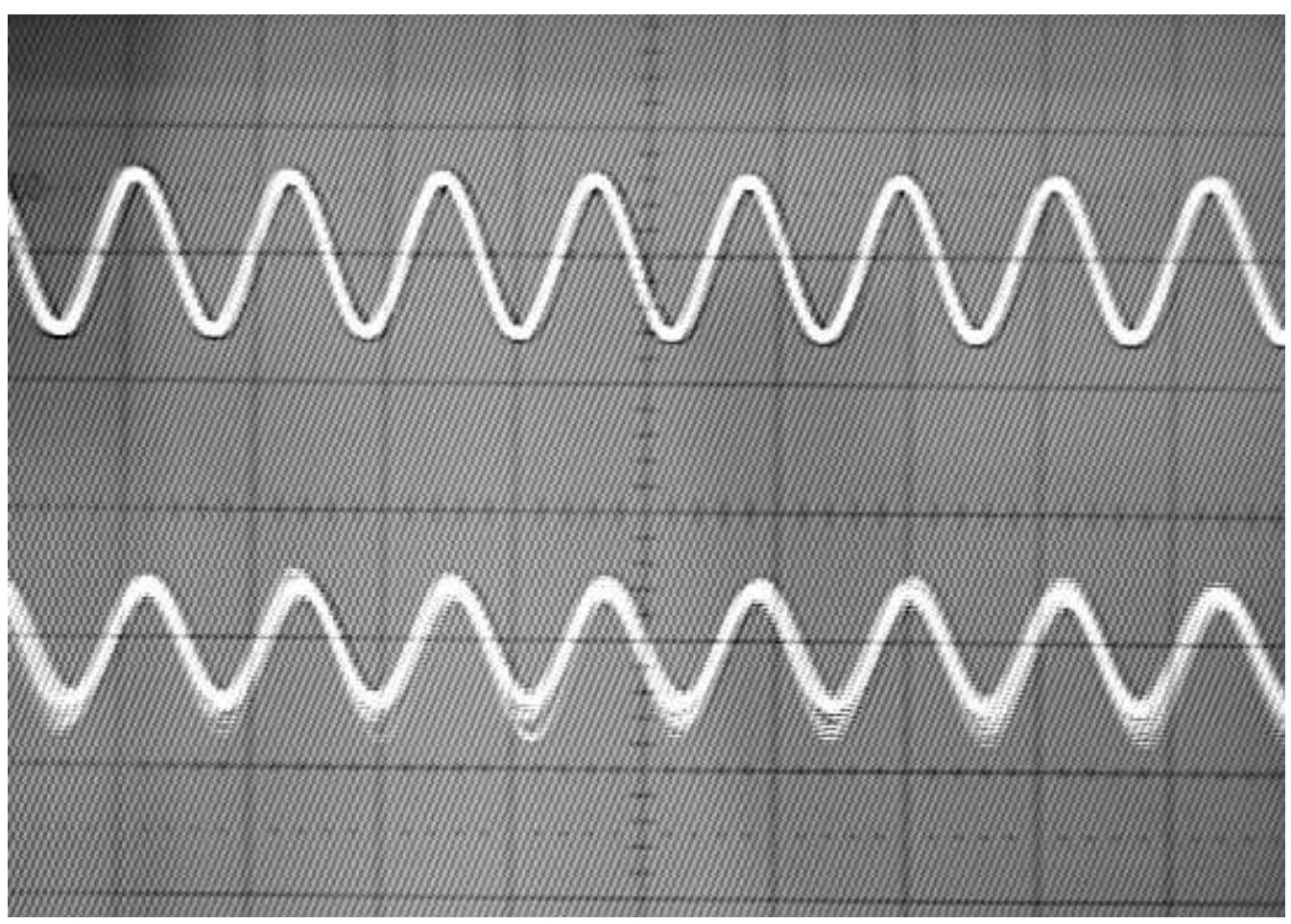

(b)

Fig. 5. (a) The transmitted signal $10^{4} f\left(V_{C 1}(t)\right)(5 \mathrm{~V} / \operatorname{div}-2 \mathrm{msn} /$ div $)$. (b) The input voltage $V_{m}(t)$ (the signal above) $(5 \mathrm{~V} / \operatorname{div}-50 \mu \mathrm{sn} / \operatorname{div})$, the recovered signal $\hat{m}(t)(1 \mathrm{~V} / \operatorname{div}-50 \mu \mathrm{sn} / \operatorname{div})$. 


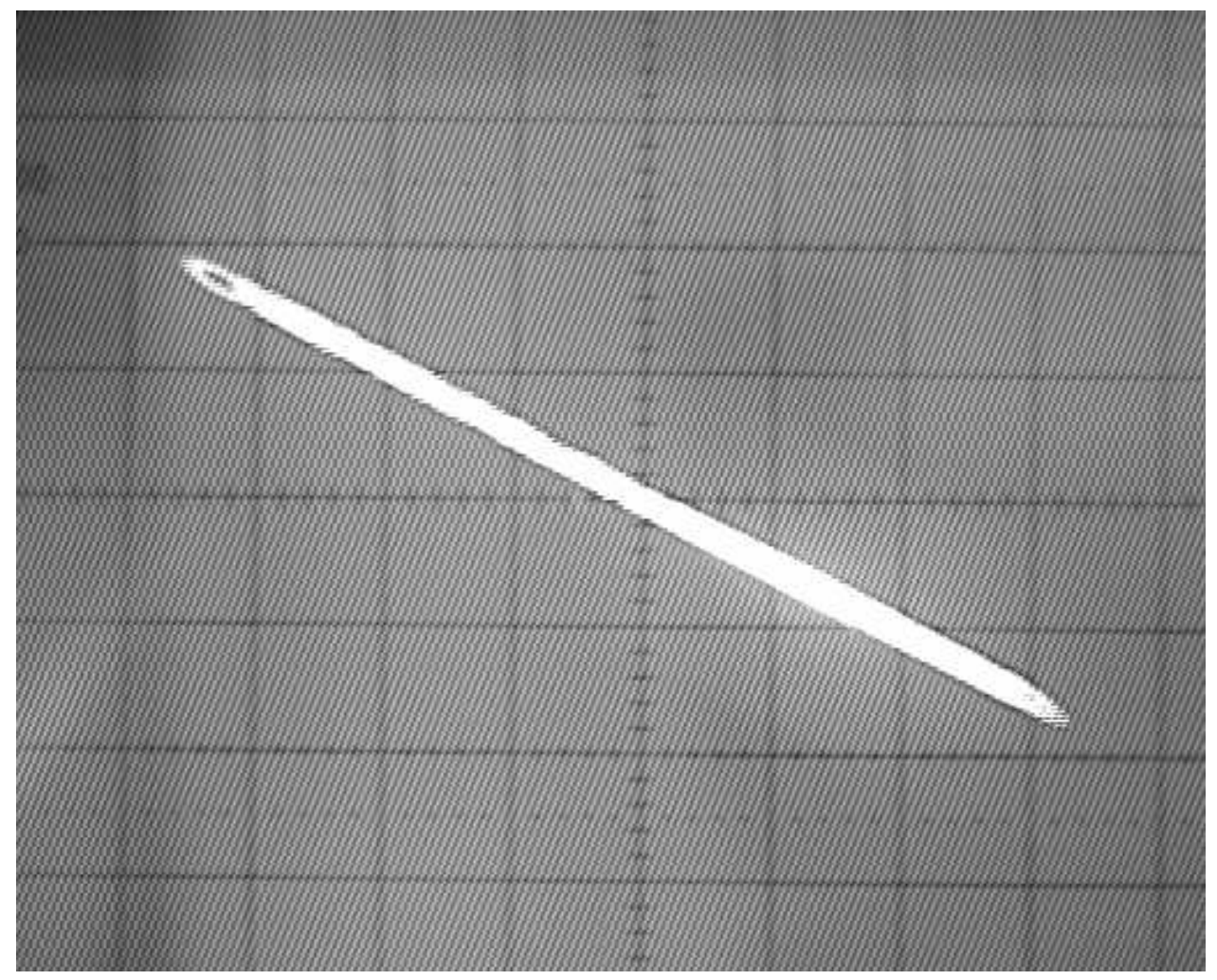

(a)

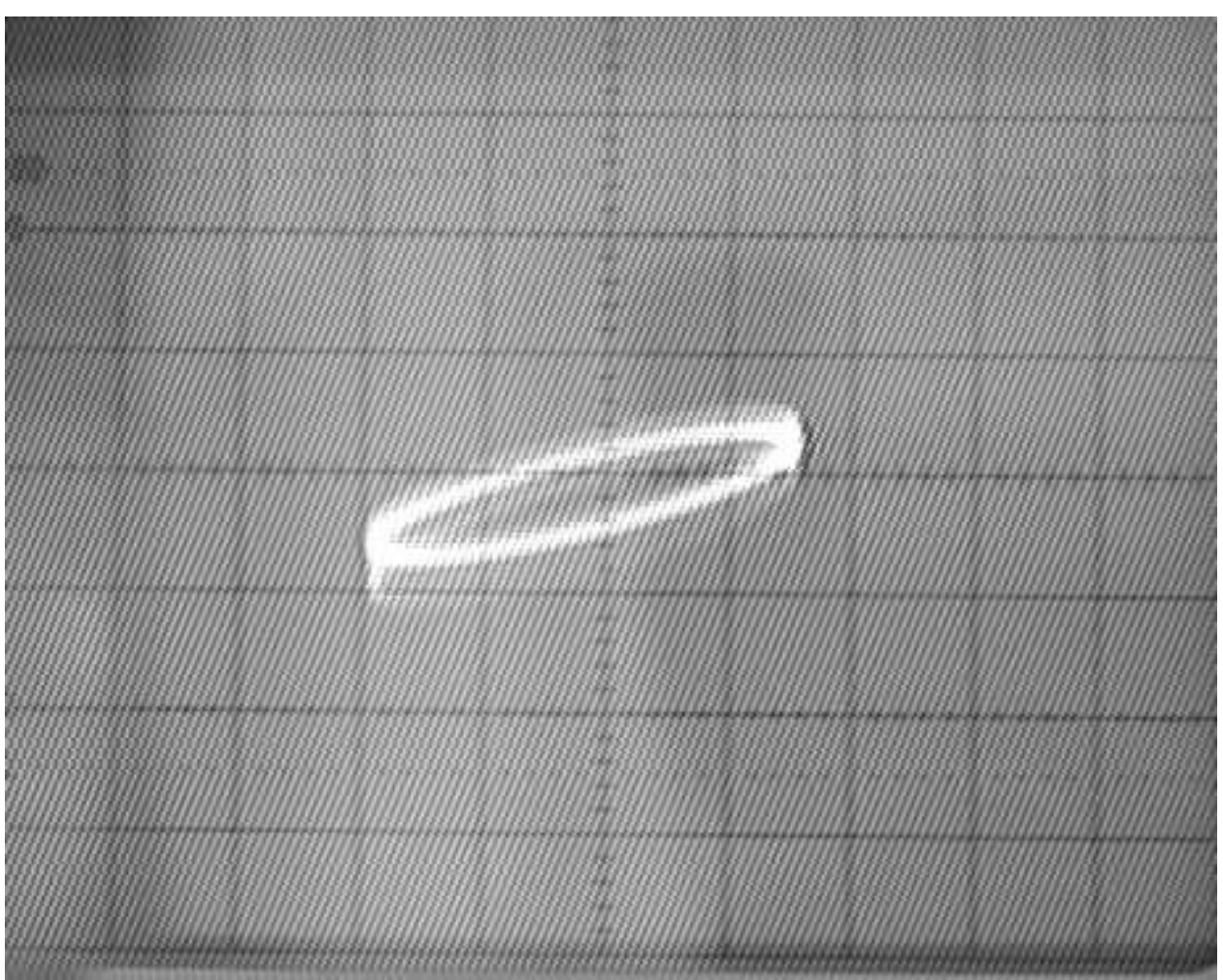

(b)

Fig. 6. (a) $V_{C 1}(t)-\hat{V}_{C 1}(t)$ plot $(2 \mathrm{~V} / \operatorname{div}-1 \mathrm{~V} / \operatorname{div})$. (b) $\hat{m}(t)-V_{m}(t)(0.5 \mathrm{~V} / \operatorname{div}-2 \mathrm{~V} / \operatorname{div})$. 


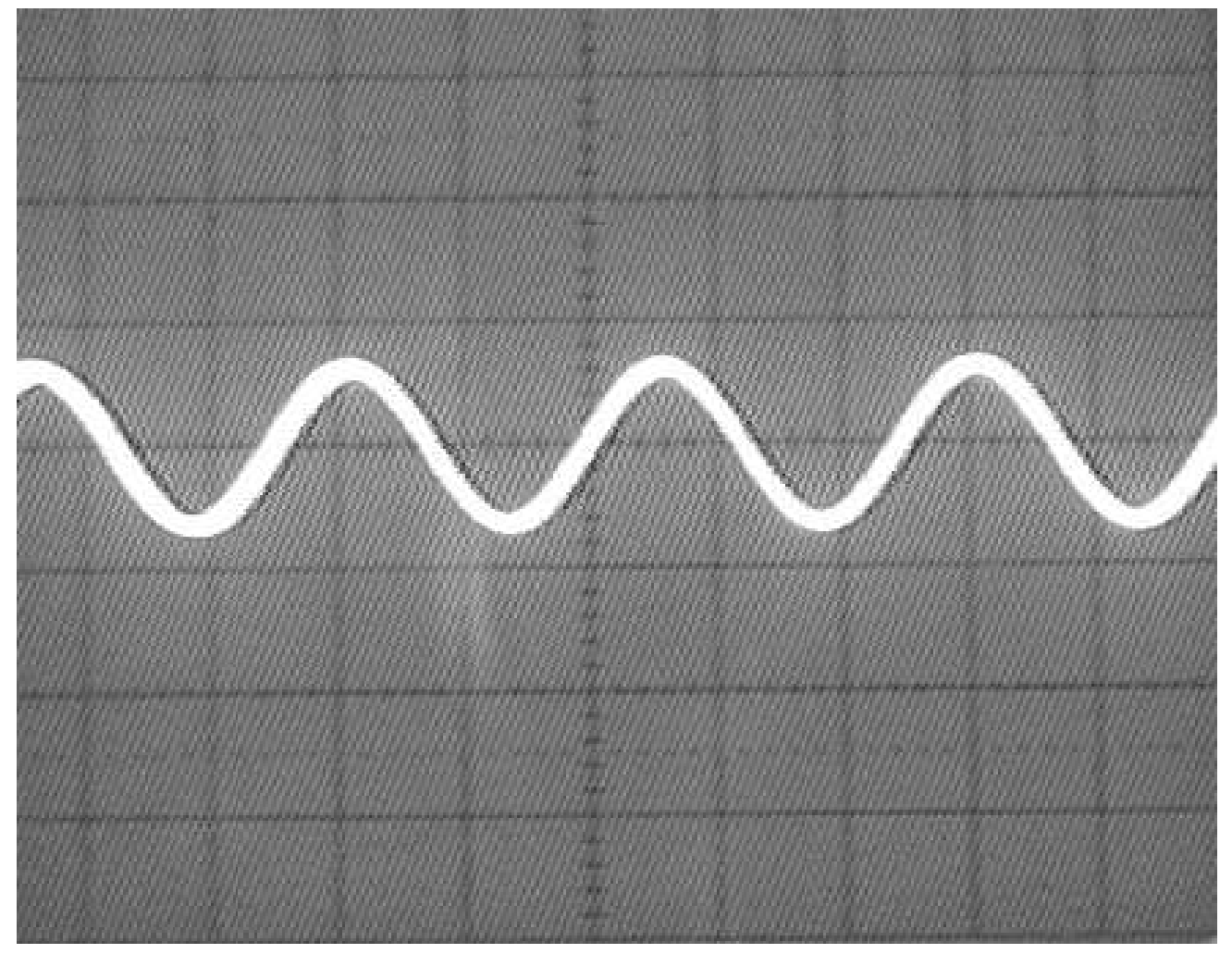

(a)

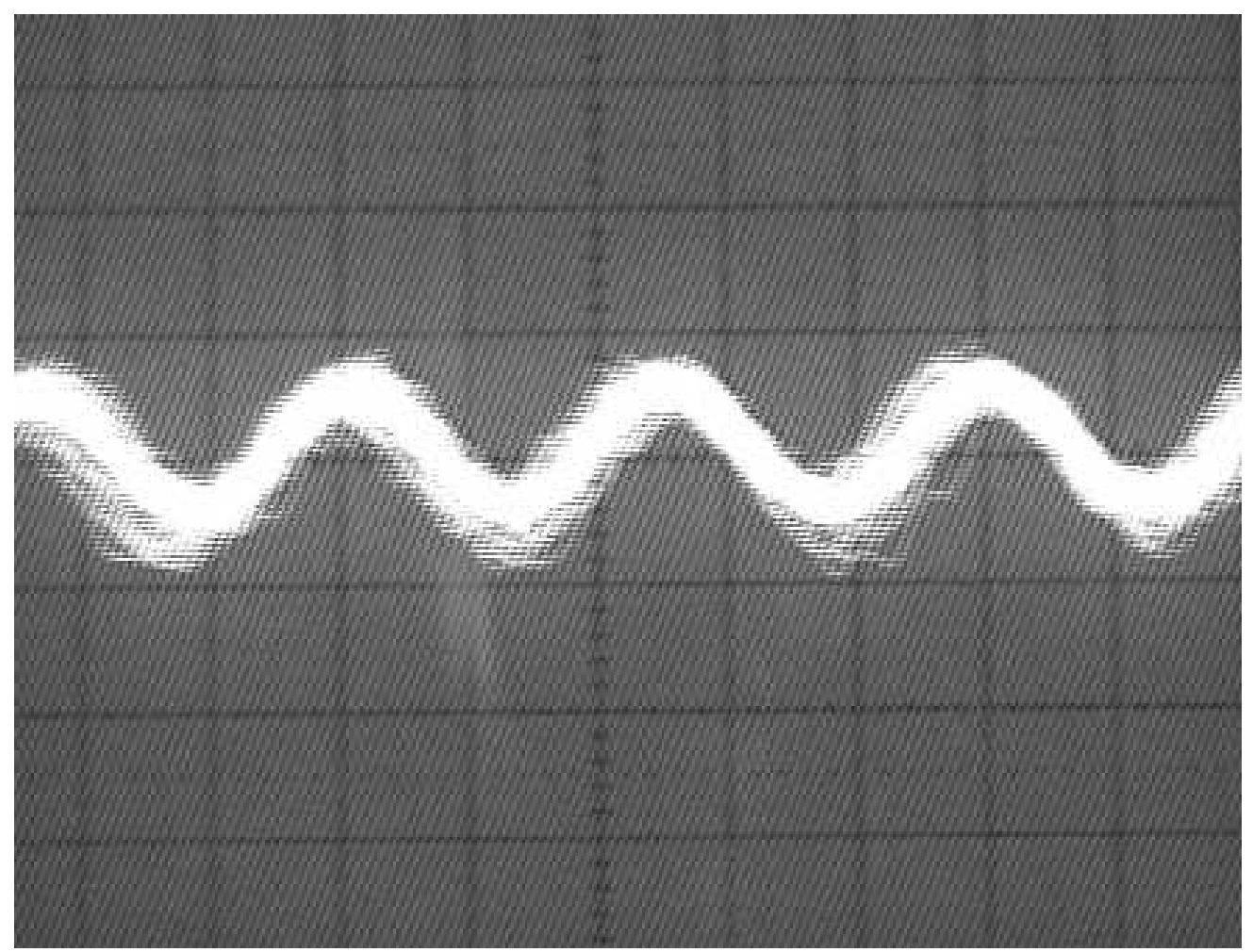

(b)

Fig. 7. (a) The input $V_{m}(t)(5 \mathrm{~V} / \operatorname{div}-20 \mu \mathrm{sn} /$ div $)$. (b) The recovered input $\hat{m}(t)$ under the channel noise effect $\left(0.5 \mathrm{~V} /\right.$ div- $^{-}$ $20 \mu \mathrm{sn} / \mathrm{div})$. 
to peak value of the transmitted signal, the $\% 23$ noise/signal ratio is obtained.

\section{Conclusion}

The presented time-domain analysis approach based on the steady-state analysis of piecewiselinear dynamic systems has yielded analytical expressions for the magnitude and phase characteristics which can be used to determine the recovered signal. These characteristics are very similar to the magnitude and phase characteristics obtained by using the harmonic balance analysis in [Savaci \& Yalçın, 1997]. The given approach does not analytically give the magnitude and frequency range for the input signal which is necessary to preserve the chaotic regimes of both Chua's circuits but the input signal's magnitude must be small compared to the amplitude of the first harmonic of the limit cycle of the double scroll regime and also the frequency of the input signal must be high compared to the fundamental frequency of the limit cycle. With such a choice for the input signal frequency, the magnitude of the forced response due to the residue term could be low, hence the input signal and the recovered signal would be very similar at the steady-state.

\section{Acknowledgment}

A part of this research has been done during Müstak E. Yalçın's stay at Istanbul Technical University.

\section{References}

Abel, A. \& Schwarz, W. [2002] "Chaos communications - principles, schemes and system analysis," Proc. IEEE 90, 691-710.

Afraimovich, V. S., Verichev, N. N. \& Rabinovich, M. I. [1987] "Synchronization of oscillations in dissipative systems," Radiophysics and Quantum Electronics (Plenum Publishing), pp. 795-803.

Anishchenko, V. C., Vadivasova, T. E., Astakhov, V. V., Sosnovtseva, O. V., Wu, C. W. \& Chua, L. O. [1995] "Dynamics of two coupled Chua's circuits," Int. J. Bifurcation and Chaos 5, 1677-1699.

Armanazi, A. N. [1973] "Steady-state analysis of piecewise linear systems with periodic inputs," Proc. IEEE 61, 789-790.

Blekhman, I. I., Landa, P. S. \& Rosenblum, M. G. [1995] "Synchronization and chaotization in interacting dynamical systems," Appl. Mech. Rev. 48, 733-752.

Carroll, T. L. \& Pecora, L. M. [1993] "Synchronizing nonautonomous chaotic circuits," IEEE Trans. Circuits Syst.-II 40, 646-650.
Carroll, T. L. [1994] "Synchronizing chaotic systems using filtered signals," Phys. Rev. E50, 2580-2587.

Chua, L. O., Komuro, M. \& Matsumoto, T. [1986] "The double scroll family," IEEE Trans. Circuits Syst.-I 33, 1072-1118.

Chua, L. O. \& Yang, L. [1988] "Cellular neural networks: Theory and applications," IEEE Trans. Circuits Syst.-I 35, 1257-1290.

Galias, Z. \& Ogarzalek, M. J. [1999] "Study of synchronized motions in a one-dimensional array of coupled chaotic circuits," Int. J. Bifurcation and Chaos 9, 2219-2224.

Güzeliş, C. [1993] "Chaotic cellular neural networks made of Chua's circuits," Chua's Circuit: A Paradigm for Chaos, ed. Madan, R. N., World Scientific Series on Nonlinear Science, Series B, Vol. 1, pp. 952-961.

Güzeliş, C. \& Chua, L. O. [1993] "Stability analysis of generalized cellular neural networks," Int. J. Circuit Th. Appl. 21, 1-33.

Hajj, I. N. \& Skelboe, S. [1981] "Steady-state analysis of piecewise-linear dynamic systems," IEEE Trans. Circuits Syst.-I 28, 234-242.

Kapitaniak, T. \& Chua, L. O. [1994] "Hyperchaotic attractors of unidirectionally-coupled Chua's circuits," Int. J. Bifurcation and Chaos 4, 477-482.

Kazantsev, V. B. \& Nekorkin, V. I. [2002] "Signal transmission in the net of two coupled electronic fibers," Proc. 10th Workshop on Nonlinear Dynamics of Electronic Systems (NDES 2002), pp. 4.9-4.12.

Kennedy, M. P. [1992] "Robust op amp implementation of Chua's circuit," Frequenz 46, 66-80.

Maistrenko, Yu. \& Popovych, O. [2000] "On strong and weak chaotic partial synchronization," Int. J. Bifurcation and Chaos 10, 179-203.

Minorsky, N. [1962] Nonlinear Oscillations (D. Van Nostrand Company, NJ).

Murali, K. \& Lakshmanan, M. [1992] "Effects of sinusoidal excitation on the Chua's circuit," IEEE Trans. Circuits Syst.-I 39, 267-270.

Nijmeijer, H. [2001] "A dynamical control view on synchronization," Physica D154, 219-228.

Pecora, L. M. \& Carroll, T. L. [1990] "Synchronization in chaotic systems," Phys. Rev. Lett. 64, 821-823.

Pecora, L. M. \& Carroll, T. L. [1991] "Driving systems with chaotic signals," Phys. Rev. A44, 2374-2383.

Pikovsky, A. S., Rosenblum, M. G., Osipov, G. V. \& Kurths, J. [1997] "Phase synchronization of chaotic oscillators by external driving," Physica D104, 219-238.

Rosenblum, M. G., Pikovsky, A. S. \& Kurths, J. [1997] "Phase synchronization in driven and coupled chaotic oscillators," IEEE Trans. Circuits Syst.-I 44, 874-881. 
Savaci, F. A. \& Yalçın, M. E. [1997] "Analysis of chaotic state modulation-demodulation system by using harmonic balance method," Proc. 13th European Conf. Circuit Theory and Design (ECCDT'97), pp. 139-143.

Suykens, J. A. K., Vandewalle, J. \& Chua, L. O. [1997] "Nonlinear $H_{\infty}$ synchronization of chaotic
Lur'e systems," Int. J. Bifurcation and Chaos 7, 1323-1335.

Yalçın, M. E., Şavkay, L. \& Savaci, F. A. [1996] "New realization of Chua's circuit by using voltage controlled voltage source (VCVS)," Proc. Int. Workshop on Nonlinear Dynamics of Electronic Systems (NDES'96), pp. 259-264. 